\title{
Comparative Study of Erbium Laser Versus A Chemo-Mechanical Caries Removal Method in Primary Teeth
}

\author{
Rana G. Salem ${ }^{*}$, Mervat I. Fawzy ${ }^{2}$, Mohammed H. Mostafa ${ }^{3}$
}

Codex : 17/21.10

azhardentj@azhar.edu.eg

http://adjg.journals.ekb.eg

DOI: $10.21608 /$ adjg.2021.20987.1207

Pediatric Dentistry \& Orthodontics ( Pediatric Dentistry, Orthodontics)

\section{KEYWORDS}

Caries removal, Papacarie,

Er,Cr:YSGG, Erbium laser.

\begin{abstract}
Purpose: The purpose of this study was to evaluate the caries removal using Er,Cr:YSGG laser and Papacarie chemo-mechanical caries removal (CMCR) method in primary teeth. Materials and Methods: Patients ( $4-8$ years old) having 40 carious primary molars with dentin involvement were selected for this study. Caries was removed using two methods; Group 1 (Laser) Waterlase MD turbo Er,Cr:YSGG Laser using Quartz tips $(\mathrm{MZ}-8)$ on the Gold handpiece and group 2 (CMCR) Papacarie Duo gel. Final restoration was done by Riva light cure Glass ionomer. Caries removal was assessed clinically by tactile and visual method (Ericson criteria). Time taken for the procedure was measured in minutes. Evaluation of pain perception was done using the Wong-Baker facial image. Microbiological evaluation was done by collecting dentin samples from the carious primary tooth before and after caries removal and total bacterial count was assessed. The restoration was evaluated using the United States Public Health Service (USPHS) at baseline, three months, six months and nine months. Results: The two groups showed a statistically significant difference (unpaired t test) in the time of treatment, Pain score and total bacterial count. Clinical evaluation of the restoration using the modified USPHS criteria showed no statistical significance between the two groups. Conclusion: Chemo-mechanical caries removal methods and laser irradiation are effective and efficient techniques for caries removal in children with high patient acceptance and no adverse effects.
\end{abstract}

\section{INTRODUCTION}

Caries removal is under continuous research to reach the most reliable and valid method. Partial removal of caries involves removal of the infected soft dentin which contains large amount of bacteria

- Paper extracted from Doctor thesis titles" Comparative Study of Erbium Laser versus a Chemo-Mechanical Caries Removal Method in Primary Teeth".

1. Assistant Lecturer of Pediatric dentistry, Faculty of Dentistry, Badr University in Cairo, Cairo, Egypt.

2. Professor of Endodontics, Faculty of Dental Medicine for Girls, Al-Azhar University, Cairo, Egypt.

3. Associate Professor of Pedodontics and Oral Health, Faculty of Dental medicine for Girls, Al-Azhar University, Cairo, Egypt.

*Corresponding author email: rana.gehad@gmail.com 
and leaving the affected dentin which is capable of remineralization and is resistant to removal. Thus to preserve the pulp vitality, removal of a minimum of the remaining sound tooth structure is advocated ${ }^{(1)}$.

For many decades, conventional mechanical cutting and drilling systems were used to remove the infected tooth structure and prepare the cavity for restoration. This process is efficient ${ }^{(2)}$ but has disadvantages such as the requirement of local anesthesia and pulp affection by heat and pressure, excessive removal of sound tooth structure and nonacceptance by the patients especially children ${ }^{(3)}$.

Laser was introduced in dentistry to overcome the shortcomings of traditional approach of caries management ${ }^{(4)}$. In the field of operative dentistry, Erbium lasers was considered promising due to their specificity in ablating enamel and dentin without side effects to the pulp and surrounding tissues provided that the appropriate parameters are employed ${ }^{(5)}$. Erbium Laser treatment is considered conservative in caries removal because it is highly absorbed in tissues with high water content as the carious tissue. In addition, the absence of noise, pressure and vibration makes the dental treatment less traumatic, especially for children. A reduced pain perception was shown in patients during laser treatment and therefore less need for local anesthesia was reported $^{(1,6)}$.

Chemo-mechanical caries removal (CMCR) is one of the most researched minimally invasive techniques. This method of caries removal involves chemical softening of carious dentin then it is removed with gentle excavation. Various chemical compositions have been introduced for chemo-mechanical caries removal since 1975 such as Caridex ${ }^{\circledR}$ and Carisolv ${ }^{\circledR}$. Recently, Papacarie ${ }^{\circledR}$ has caught the interest of various clinicians because of its advantages over other products ${ }^{(3)}$. Increased patients' compliance, avoiding the use of local anesthesia and tissue preservation are the main advantages of CMCR agents ${ }^{(7)}$. This study will compare two minimally invasive techniques; Laser and CMCR in terms of their efficiency, efficacy, patient acceptance and antibacterial effect to determine which method can be successfully used with young patients.

\section{MATERIALS AND METHODS}

This research was authored as stated in the CONSORT (Consolidated Standards of Reporting Trials) statement.

\section{Patient selection:}

Patients (4-8 years old) were selected for this study. Children were diagnosed, selected, treated and followed up in the outpatient clinic of Pedodontics and Oral Dental Health department, Faculty of Dental medicine for Girls, Al -Azhar University. All children were free of any systemic diseases, hereditary anomalies or any antibiotic medication during the study.

\section{Ethical consideration}

Ethical clearance was obtained in accordance with guidelines from research ethics committee approval of Dental Medicine Al-Azhar University for the study. An informed consent was signed by the parent before the procedure.

\section{Randomization:}

Regulated by the type of caries removal method, the primary molars were branched randomly and equally into two groups using envelopes simple randomization as following: Group 1: 20 carious primary molars, Caries removal by Er,Cr:YSGG Laser (Waterlase MD turbo, Biolase).

Group 2: 20 carious primary molars, Caries removal by CMCR (Papacarie ${ }^{\circledR}$ Duo) according to manufacturer's instructions.

\section{Inclusion Criteria:}

Criteria included patients with 40 carious primary molars with dentin involvement ${ }^{(8)}$. Carious lesions were open and easily accessible for hand instrumentation ${ }^{(3)}$. Teeth were asymptomatic with no clinical evidence of pulp pathosis ${ }^{(8)}$. 


\section{Caries removal procedure:}

Introduction of the child to the dental unit and behavioral modification was done using Tellshow-do method. No local anesthesia was used to avoid altering the pain perception of the child. A preoperative dentin sample was taken with a sharp excavator and immediately transferred into a screw cap vial containing transport medium. Relative isolation using cotton rolls and aspirator were used.

\section{Group 1 (Laser):}

This group was composed of 20 primary molars in which the caries was removed using Waterlase MD turbo Er,Cr:YSGG Laser (Biolase). Quartz tips (MZ - 8) was used on the Gold handpiece.

Operating parameters: Wavelength $2780 \mathrm{~nm}$, Power output $3 \mathrm{~W}^{(9)}$, Mode: $\mathrm{H}$ mode, Frequency: $15 \mathrm{~Hz}$, Energy Value: < $150 \mathrm{~mJ}{ }^{(10)}$, Water $50 \%$, Air $80 \%$ as recommended by the manufacturer.

Procedure: A distance of $1.5 \mathrm{~mm}$ was maintained between the Fiber Tip and the cavity while moving the Handpiece gently in a circular, brushing, or inand-out motion, as required, over the tissue surface as required.

\section{Group 2 (CMCR):}

This group was composed of 20 primary molars in which the caries was removed according to the manufacturer's instructions using Papacarie ${ }^{\circledR}$ Duo gel (Formula \& Acao (F\&A Laboratorio Farmaceutico LTDA). San Pablo, Brasil).

Procedure: The carious cavity was filled with Papacarie $^{\circledR}$, and the gel was left undisturbed for 30 seconds in acute caries and 40-60 seconds in chronic caries. After degradation of the collagen, oxygen was freed and bubbles appeared on the surface which caused blurring and turbidity of the gel and indicated that its removal could be started by gently scrapping with a spoon excavator in a pendulum movement with no pressure. Additional fresh gel was applied and excavated until the gel is no longer cloudy. Reapplication of the gel as many times as necessary was done until a light color was observed.
The fresh gel is blue and clear, but after its application, the gel denatured the non-structured collagen fibers of the carious lesion and become turbid and dark in color. The cavity was wiped by a moist cotton pellet and rinsed.

Checking of the cavity for complete caries removal was done using the Ericson et al criteria ${ }^{(11)}$ that a sharp explorer should not stick in the dentine and not give a 'tug back' sensation. A postoperative dentin sample was taken and put in the screw cap vial with transport medium. The cavity was prepared for final restoration by conditioning for 10 seconds by Ketac $^{\mathrm{TM}}$ Conditioner. Glass ionomer capsule Riva light cure was applied using the Glass ionomer gun and a ball burnisher and light cured.

\section{Assessment parameters}

\section{Evaluation of caries removal:}

It was assessed clinically by tactile and visual method (Ericson et al. criteria ) of caries detection with the help of mouth mirror and explorer that does not stick to dentine and not give a tug back sensation $^{(11)}$.

\section{Evaluation of the time required for caries removal:}

Time taken for the procedure was measured in minutes from the start of caries removal till the cavity was confirmed to be caries free using a digital stopwatch.

\section{Evaluation of pain perception:}

Caries removal was done without the use of local anesthesia. Indication of the degree of pain felt was done by the patient using Wong-Baker facial image scale $^{(12)}$. (Fig. 1)

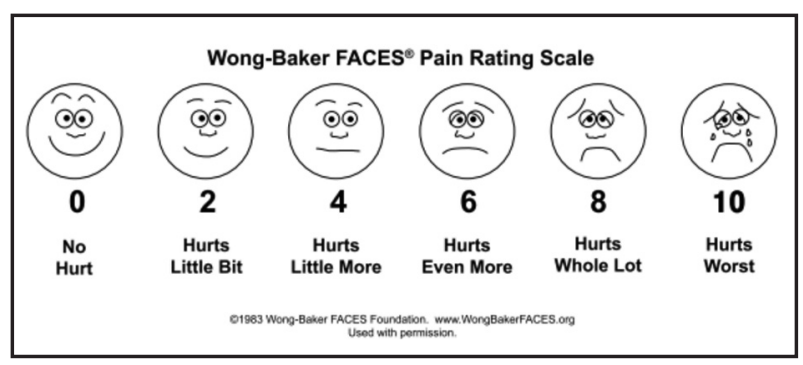

Figure(1) showing Wong-Baker FACES Pain Rating Scale 


\section{Microbiological evaluation:}

Samples were collected from the carious primary tooth before and after caries removal. Under aseptic precautions microbial samples were taken from the participants using sterile excavators and transferred to a sterile screw cap vials containing $2 \mathrm{ml}$ of Thioglycolate medium and transported to the laboratory within 1 hour of collection for further processing.

A serial dilution was made from the dentin sample. A volume of $0.1 \mathrm{~mL}$ was spread onto each of the Brain heart infusion (BHI) plates. The plates of (BHI) were incubated aerobically for 48 hours at $37^{\circ} \mathrm{C}$. The count from each plate was interpreted as colony forming unit (CFU).

\section{Evaluation of restoration:}

The restoration was evaluated using the USPHS criteria for direct clinical evaluations of restorations. The color match, marginal discoloration, secondary caries, Anatomic contour, Marginal integrity, Surface texture and Gross fracture were recorded at baseline, three months, six months and nine months ${ }^{(13)}$.

\section{Statistical analysis}

Values were presented as mean and standard deviation (SD) values. Time, pain and bacterial count results were explored for normality using Kolmogorov-Smirnov test of normality. The results of Kolmogorov-Smirnov test indicated that most of data were normally distributed (parametric data), so paired (dependent) $t$ test was used to compare pre and post values; while unpaired test was used to compare the 2 groups. Qualitative data as the clinical evaluation criteria were compared using chi square test. Statistical analysis was performed with SPSS 19.0 (Statistical Package for Scientific Studies, SPSS, Inc., Chicago, IL, USA) for Windows.

\section{RESULTS}

A higher mean time $(8.18 \pm 1.56)$ was recorded in CMC group, in comparison to $(6.27 \pm 1.01)$ in laser group. Unpaired t test revealed that the difference between groups was statistically significant $(\mathrm{p}=0.00)$ (Fig. 2).

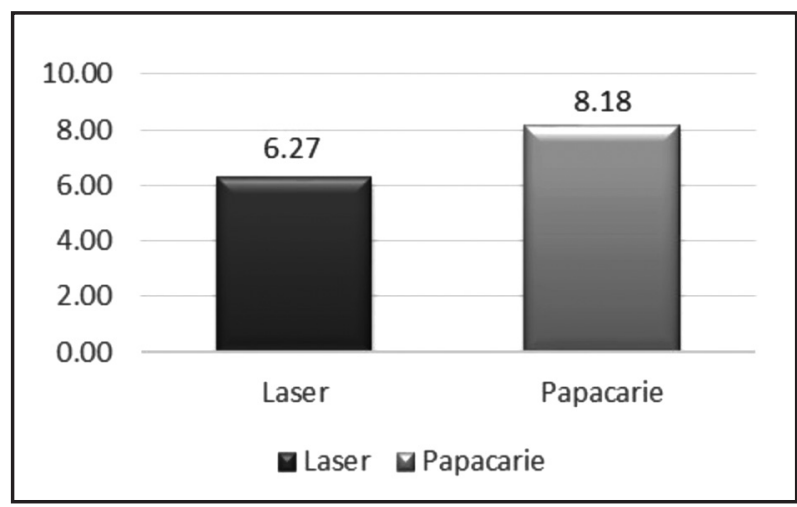

Figure (2) Bar chart illustrating mean time of treatment in minutes in both groups

A higher mean of pain score $(1.27 \pm 0.45)$ was recorded in laser group, in comparison to $(0.57 \pm 0.19)$ in CMC group. Unpaired t test revealed that the difference between groups was statistically significant $(\mathrm{p}=0.00)$ (Fig. 3).

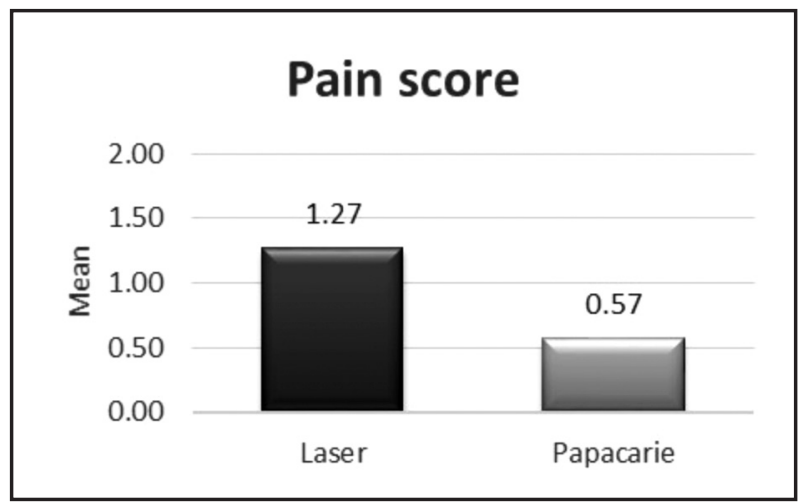

Figure (3) Bar chart illustrating mean pain score in both groups

Regarding difference of bacterial count due to treatment, a higher mean decrease $(-3.22 \pm 0.39)$ was recorded in laser group, in comparison to $(-2.48 \pm 0.8)$ in CMC group. Unpaired $t$ test revealed that the difference between groups was statistically 
significant ( $\mathrm{p}=0.0006$ ) (Figure 4). Regarding percent change of bacterial count due to treatment, a higher mean percent decrease $(-46.83 \pm 5.08)$ was recorded in laser group, in comparison to $(-32.37 \pm 8.78)$ in $\mathrm{CMC}$ group. Unpaired $\mathrm{t}$ test revealed that the difference between groups was statistically significant ( $\mathrm{p}=0.00)$ (Fig. 4).

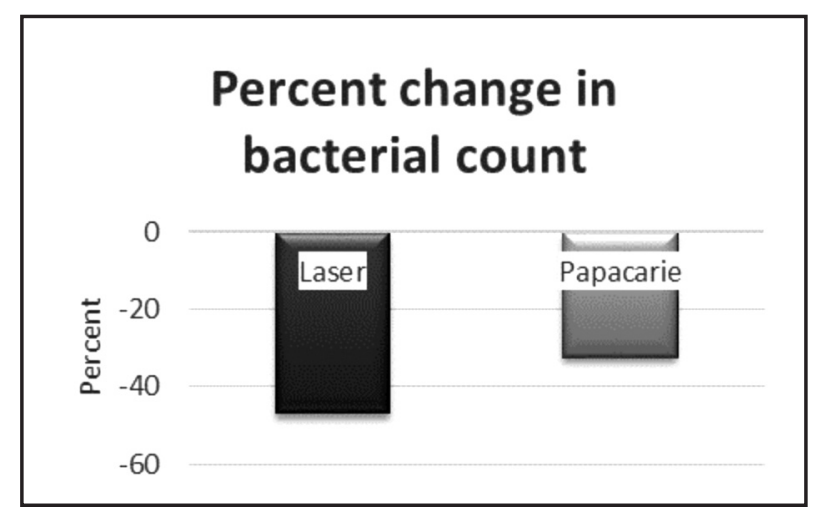

Figure (4) Bar chart illustrating mean percent change in bacterial count in both groups.

Clinical evaluation of the restorations was done using the modified USPHS criteria showed no statistical significance between the two groups regarding the color match, marginal discoloration, secondary caries, Anatomic contour, Marginal integrity, Surface texture and Gross fracture.

\section{DISCUSSION}

The concept of minimally invasive dentistry has evolved because we now have a better understanding of the carious disease as we can intercept its development and have the technical facilities to make smaller fillings and remove a minimal amount of healthy tooth substance ${ }^{(4)}$. Minimally invasive dentistry includes different techniques such as air abrasion, atraumatic restorative technique, sono-abrasion, laser and chemo-mechanical caries removal methods ${ }^{(14)}$.

A clinical comparison between Papacarie ${ }^{\circledR}$ and Er,Cr:YSGG laser irradiation in their efficacy, efficiency, anti-bacterial effect, pain score and follow up of the restoration for 9 months was done.
Er,Cr:YSGG (wavelength: $2780 \mathrm{~nm}$ ) is considered ideal for pediatric dentistry since they have high affinity to water and shallow depth of tissue penetration. Children are comfortable during treatment due to the lack of vibratory sensation and the water spray from the non-contact tip which avoids generating an excessive amount of heat and pain during laser application ${ }^{(2)}$. In addition many studies have reported that lasers produce a mild local anesthetic effect ${ }^{(16)}$.

Laser parameters used in this study were adjusted to power output of $3 \mathrm{~W}$ with $80 \%$ air and $50 \%$ water for dentine in accordance to similar studies ${ }^{(2)}$. Quartz tips of diameter $0.8 \mathrm{~mm}$ was used (MZ 8) in a gold handpiece.

Papacarie claims to selectively remove caries. It is made of papain, chloramines and toluidine blue. It has antibacterial and anti-inflammatory characteristics. Papain targets only infected tissues due to the absence of plasmatic anti-protease called alpha- 1 anti-trypsin that is present in sound tissues only. Toluidine blue also is an antimicrobial agent ${ }^{(14)}$.

Several evaluation parameters were used to compare the two minimally invasive techniques to remove caries in children. To assess the efficacy of caries removal, the most widely used clinical criterion was adopted, where a moderately sharp explorer did not stick in the remaining dentine known as the visual and tactile sensation criteria by Ericsson ${ }^{(11)}$. These criteria were chosen as it is the method commonly used by operators in their practice and was found to be reliable, valid and clinically proven.

Other aids to assess caries removal as caries detector dyes were not used as none of the available is caries specific, it does not discriminate between diseased and healthy tooth structures in addition to the fact that it stains normal dentin at the amelodentinal junction also it stains food debris, enamel pellicle and any other organic matter trapped in occlusal fissures $^{(15)}$.

Laser was reported to have lower efficiency of caries removal than conventional bur preparation as the treatment must be interrupted to continually 
check the presence of remaining infected dentin since the laser has decreased tactile sensitivity and it does not curve making the preparation more difficult while in conventional bur preparation the haptic feedback allows the operator to stop the treatment when finished ${ }^{(1)}$. In the present study we sought to preserve unsupported enamel according to the minimally invasive dentistry concept so complete removal of carious dentin from the surrounding walls that was missed by laser caries removal was done using a sharp excavator ${ }^{(1)}$.

Using the Papacarie gel, multiple reapplications was done until it remained unchanged in color indicating that no soft carious dentin was left. Chemo-mechanical techniques are effective in cavities where good access to dentine caries can be obtained but it is not effective for pits and fissures caries where air rotor is required ${ }^{(17)}$. In our study, final restoration was not applied until after complete caries removal was achieved.

The time taken for complete caries removal was recorded to assess the efficiency of the caries removal method. High heterogeneity in the time needed was observed due to multiple factors such as the age of the patient and the site, area and stage of the carious lesion in addition to the longer treatment time for children than for adults ${ }^{(4)}$.

During caries removal using Laser, the mean of the preparation time was 6.27 minutes which was in accordance to other studies ${ }^{(2)}$. A systematic research in 2017 compared the Erbium laser technology versus the traditional methods, all studies showed less preparation time using the conventional methods than the Erbium laser ${ }^{(4)}$. Laser irradiation time could be shorter by increasing the irradiation energy but this would cause thermal damage to the tooth structure, a discomforting vibration and increased pain sensation ${ }^{(16)}$.

Using the Papacarie gel for caries removal was reported to be a slower method which might be because of it needs to be applied multiple times until complete caries removal was achieved ${ }^{(2,16)}$. The mean time for caries removal using Papacarie in this study was 8.18 minutes.

In comparison, a higher mean time $(8.18 \pm 1.56)$ was recorded in CMCR group, in comparison to $(6.27 \pm 1.01)$ in laser group. A statistically significant difference was observed between the two groups.

For children, pain is a more concerning than the treatment time as it may lead to uncooperative behavior. $^{(2)}$

Dentists percept what the pain must be like. However, the patient is the only one who can tell what it is like. Hence, to assess pain effectively, reliable and valid assessment scales that do not depend on verbal descriptions are needed especially in children so that modification of the treatment technique and parameters can be done according to the intensity of pain experienced by the patient. Many scales are used to assess pain as visual analogue scale $(\mathrm{VAS})^{(2,11)}$, FLACC scale (face, leg, activity, cry, consolability scale) ${ }^{(16)}$ and SEM scale (sound, eye and motor scale) ${ }^{(3)}$.

Our study used the Wong Baker scale (WBS) which is considered to have many advantages for use in children. Its use and rating is easy, the faces are understandable and not time consuming. It is reliable and valid for ages ranging from 3 to 18 years ${ }^{(12,15)}$.

The present study results showed a higher mean of pain score $(1.27 \pm 0.45)$ in laser group, in comparison to $(0.57 \pm 0.19)$ in CMCR group. Unpaired $t$ test revealed a statistically significant difference between groups. In cavities reaching dentin, children showed no or mild pain response while in deeper parts of the dentin a moderated response of pain was expressed. These results were in accordance with several studies also using Er,Cr:YSGG laser in children12 18 20. A study comparing the laser preparation to the conventional preparation reported laser to be more comfortable in $70 \%$ of the children than the mechanical preparation ${ }^{(2)}$, another study reported that $68 \%$ of the cases felt no pain at all ${ }^{(18)}$. 
A review of literature done in 2017 comparing the Erbium laser treatments to the conventional methods of cavity preparations reported that laser is more comfortable for patients, fewer patients required local anesthesia suggesting that caries removal using laser increase the patients' subjective acceptance to treatment since it can significantly reduce pain ${ }^{(4)}$. The lack of vibration and noise associated with the typical drill is found in the laser system due to the non-contact mode of operation which makes it more agreeable to the in addition to the mild local anesthetic effect which allows for shallow cavity preparations to be completed without local analgesia ${ }^{(11)}$. The transient anesthetic effect is produced by blocking nerve conduction at $\mathrm{Na} / \mathrm{K}$ pump, disruption of nerve terminals in the dentinal tubules, disruption of the myelin sheath in the pulp and the degeneration of nerve terminals between the odontoblasts ${ }^{(16)}$. Moreover, most of the energy of the erbium laser is absorbed by water and it has a shallow penetration force ${ }^{(4)}$.

With respect to CMCR methods, a low pain score was obtained owing to selective removal of only infected dentin whereas cutting in sound dentine often results in some level of pain. Also, the gel covers the cavity during the procedure which may have a thermal insulating function. In addition to lowering the child's anxiety when told that the drill may not be used ${ }^{(16)}$.

Total bacterial count was assessed by obtaining dentin samples before and after caries removal. Both caries removal methods led to reductions in the total bacterial count that are statistically significant which indicates the efficacy of both methods. A higher mean decreases in the percent change of bacterial count $(-46.83 \pm 5.08)$ was recorded in laser group, in comparison to $(-32.37 \pm 8.78)$ in CMC group.

In the laser group, the cell structure of the bacteria can be modified by the increased temperature during ablation of the dentin leading to reduction in the bacterial viability ${ }^{(1)}$. The Papacarie gel has antibacterial properties which affecting the number of microorganisms found in the dentin following the removal of the carious tissue. The reduction of the total bacterial count is in agreement with findings described in previous study comparing Papacarie with the traditional method of caries removal ${ }^{(19)}$.

Resin modified glass ionomer was used as a final restoration. This material is biocompatible, bonds chemically to the tooth structure and releases fluoride but compared with resin composites, glass ionomers have higher early wear and are less aesthetic. High viscosity glass ionomer have reported no difference in survival rates ${ }^{(20)}$.

Our study used the Light Cure Riva glass ionomer restoration and was followed up for 9 months. The USPHS criteria proposed by Ryge was used ${ }^{(13)}$ which considers surface texture, marginal adaptation, discoloration, anatomic form, color match and secondary caries.

No statistically significant difference was found between the laser group and the CMCR group in accordance to a study comparing Laser preparation to bur preparation ${ }^{(1)}$.

The ability of glass ionomer cements to prevent caries lesions in the margins of occlusal and occlusoproximal restorations in primary teeth was evaluated in a meta-analysis and compared with that of other restorative materials and was found to be associated with better ability to prevent carious lesions ${ }^{(21)}$.

Based on the findings of the present study, chemo-mechanical caries excavation and laser irradiation follow the principles of minimally invasive dentistry. Papacarie and Er,Cr:YSGG laser achieved significant reduction in the total bacterial count with comparable effectiveness. Both techniques showed a low pain score and no need for use of local anesthesia. Preparation time with laser was longer and restorations following caries removal by both methods showed excellent results after 9 months follow up.

A limitation facing this study was the tooth selection criteria which was a cavity with dentin caries that is open for hand instrumentation. Many of the teeth which conformed with this criterion were symptomatic and indicated for pulp therapy. 


\section{CONCLUSION}

Chemo-mechanical caries removal methods and laser irradiation are effective, efficient and effective techniques for caries removal in children with high patient acceptance and no adverse effects which makes their use a successful alternative for conventional cavity preparation in children.

\section{REFERENCES}

1. Valério RA, Borsatto MC, Serra MC, Polizeli SAF, Nemezio MA, Galo R, et al. Caries removal in deciduous teeth using an Er:YAG laser: a randomized split-mouth clinical trial. Clin Oral Investig. 2016;20:65-73.

2. Eren F, Altinok B, Ertugral F, Tanboga I. The effect of erbium, chromium:yttrium-scandium-gallium-garnet (Er,Cr:YSGG) laser therapy on pain during cavity preparation in paediatric dental patients: a pilot study. Oral Health Dent Manag. 2013;12:80-4.

3. Nagaveni N, Radhika N, Satisha T, Ashwini K, Neni S, Gupta S. Efficacy of new chemomechanical caries removal agent compared with conventional method in primary teeth: An in vivo study. Int J Oral Heal Sci. 2016;6:52-8.

4. Tao SS, Li L, Yuan H, Tao SS, Cheng Y, He L, et al. Erbium Laser Technology vs Traditional Drilling for Caries Removal: A Systematic Review with Meta-Analysis. J Evid Based Dent Pract. 2017;17:324-34.

5. de Almeida Neves A, Coutinho E, Cardoso MV,Lambrechts P, Van Meerbeek B. Current concepts and techniques for caries excavation and adhesion to residual dentin. J Adhes Dent. 2011;13:7-22.

6. Bona A, de Azevedo CS, da Ana PA, Brossi S, Maria D. Laser Technology for Caries Removal. Contemp. Approach to Dent. Caries, vol. 2, IntechOpen; 2012, p. 291-312.

7. Ramamoorthi S, Nivedhitha M, Vanajassun Pp. Effect of two different chemomechanical caries removal agents on dentin microhardness: An in vitro study. J Conserv Dent. 2013;16:429-33.

8. Aswathi K, Rani P, Athimuthu A, Prasanna P, Patil P, Kj D. Comparison of efficacy of caries removal using polymer bur and chemomechanical caries removal agent : A clinical and microbiological assessment - An in vivo study. J Indian Soc Pedod Prev Dent. 2017;35:6-13.
9. Fatma Dilsad O, Ergin E, Attar N, Gurgan S. Comparison of laser- and bur-prepared class I cavities restored with two different low-shrinkage composite resins: a randomized, controlled 60-month clinical trial. Clin Oral Investig. 2020;24:357-68.

10. Olivi G, Olivi M. Lasers in Restorative Dentistry. Springer Berlin Heidelberg; 2015.

11. Ericson D, Zimmerman M, Raber H, Götrick B, Bornstein R, Thorell J. Clinical Evaluation of Efficacy and Safety of a New Method for Chemo-Mechanical Removal of Caries. Caries Res. 1999;33:171-7.

12. Boj J, Galofre N, Espana A, Espasa E. Pain Perception in Pediatric Patients Undergoing Laser Treatments. J Oral Laser Appl. 2005;5:85-9.

13. Barnes DM, Blank LW, Gingell JC, Gilner PP. A clinical evaluation of a Resin-Modified Glass Ionomer restorative material. J Am Dent Assoc. 1995;126:1245-53.

14. Bhardwaj A, Bhardwaj S V. Papacarie $\AA^{\circledR}$ containing papain: A Natural Chemomechanical Caries Removal Agent. Res J Pharm Biol Chem Sci. 2012;3:660-6.

15. McComb D. Caries-detector dyes - How accurate and useful are they? J Can Dent Assoc. 2000;66:195-8.

16. Polonsky M, Gutknecht N, Franzen R. Review of possible predictors for pain perception with class 1-5 cavity preparations using Er,Cr:YSGG laser: a retrospective clinical in vivo study. Lasers Dent Sci. 2017;1:9-21.

17. Bohari MR, Chunawalla YK, Mohammed B, Ahmed N Clinical Evaluation of Caries Removal in Primary Teeth using Conventional, Chemomechanical and Laser Technique: An in vivo Study. J Contemp Dent Pr. 2012;13:40-7.

18. Deng Y, Feng GE, Hu BO, Kuang Y, Song J. Effects of Papacarie on children with dental caries in primary teeth : a systematic review and meta-analysis. Int J Paediatr Dent 2018;28:361-72.

19. Boj JR. The Future of Laser Pediatric Dentistry. J Oral Laser Appl. 2005;5:173-8.

20. Matsumoto K, Hossain MMI, Kawano H, Kimura Y. Clinical Assessment of Er , Cr : YSGG Laser Application for Cavity Preparation. J Clin Laser Med Surg. 2002;20:17-21.

21. Motta LJ, Bussadori ISK, Campanelli IAP, Andre II, Silva L, Alfaya TA, et al. Efficacy of Papacarie in reduction of residual bacteria in deciduous teeth: a randomized, controlled clinical trial. Clinics. 2014;69:319-22. 